\title{
Introduction à l'analyse de chimères technologiques, le cas du vote électronique
}

\author{
Chantal Enguehard \\ LINA - UMR CNRS 6241 \\ 2, rue de la Houssinière, BP 92208, 44322 Nantes Cedex 03 - France \\ chantal.enguehard@univ-nantes.fr
}

Les chimères, issues des temps médiévaux, semblaient n'avoir survécu que sur les blasons. Ces êtres mythiques, souvent représentés sous la forme d'un être à corps de lion et tête et buste de femme, sont devenus l'archétype des créatures dont l'existence est impossible. Ce sens est si prégnant que sa dérivation adjectivale "chimérique" a pris de sens d'"invraisemblable". Assemblages inédits de fragments de réalité incompatibles, les chimères ré-apparaissent sous de nouvelles formes.

L'apparition du vote électronique en France coïncide avec le nouveau millénaire ${ }^{1}$ et la vulgarisation de l'usage de l'informatique. Une décennie plus tard, son développement pénètre toutes les sphères de la société, depuis les votes politiques dans les mairies jusqu'à l'organisation de primaires dans les partis en passant par le Conseil de l'Ordre des Infirmières, le Comité National de la Recherche Scientifique ou encore le CNRS qui a procédé au renouvellement des représentants du personnel au sein de son Conseil d'Administration par un vote par internet en 2009.

La transformation des objets nécessaires à l'exercice du devoir électoral y est radicale : il n'y a plus d'urne, les bulletins de vote sont dématérialisés, les scrutateurs disparaissent. La rapidité de ce changement contraste avec la prudence des transformations précédentes : il a fallu plus de quarante ans de propositions et de discussions pour que l'usage d'un isoloir et d'une enveloppe devienne obligatoire en France (en 1913), et l'urne n'est devenue transparente qu'en 1988 [Offerlé 2002].

Cette célérité dénote une approche superficielle de l'acte de voter, oublieuse de son caractère profondément multidimensionnel. Les dispositifs de vote électronique ont été spécifiés en appliquant uniquement les méthodes usuelles pour la conception de systèmes de traitement de données, sans prendre en compte les dimensions éthiques, sociales, politiques et juridiques de l'acte de voter. Cette vision restreinte a eu pour conséquence la conception et la diffusion de systèmes insatisfaisants. En effet, une analyse tenant compte de toutes les dimensions des scrutins permet de conclure à l'impossibilité, en l'état actuel de la science, de concevoir des systèmes informatiques recueillant les voix des électeurs et effectuant le dépouillement tout en respectant le droit de regard et de contrôle des électeurs sur les opérations électorales.

Pourtant, des systèmes de vote électroniques existent, sont commercialisés et utilisés dans un cadre légal. Ces systèmes apparaissent comme des chimères à double titre : il s'agit d'objets impossibles qui doivent leur existence à une conception des scrutins réduite à leur dimension technique ; ces objets sont porteurs de nombre de fantasmes cybernétiques (facilitation de l'acte de voter, augmentation de la participation, etc.).

Nous illustrerons cette thèse de trois exemples : l'encadrement juridique des machines à voter en France, le concept de vote avec trace "papier", et le cas du vote par internet. Tout au long de

1 France Télécom et le Ministère de l'Intérieur sont partenaires du projet européen e-poll ; avec EADS, la France est leader de cybervote (Projet du $5^{\circ}$ PCRD IST-1999-20338)

Enguehard, C. Introduction à l'analyse de chimères technologiques, le cas du vote électronique. Cahiers Droit Sciences et Technologie, n³, p.261-278, CNRS editions, 2010. ISBN 978-2-271-06974-0. SSN 1967-0311. 
l'exposé nous présenterons quelques conséquences de la transformation des objets du vote quant à leurs propriétés physiques et légales.

\section{1 - Objets}

\section{1 - Scrutins}

Le but d'un scrutin est de désigner un candidat parmi plusieurs possibilités présentées aux électeurs. Certains scrutins visent à élire des représentants physiques (élections), d'autres à prendre des décisions en acceptant ou en rejetant une proposition (référendums).

Cet article examine le déroulement de la procédure électorale pendant la durée de vote, depuis l'ouverture des bureaux de vote jusqu'au décompte des voix sans se limiter à son aspect calculatoire (additionner les voix), mais en prenant en compte plusieurs de ses dimensions.

\section{— dimension éthique}

Le droit de vote fait partie des libertés fondamentales dont jouit tout citoyen majeur non déchu de ses droits civiques. Chaque électeur dispose d'une voix et a le droit d'exprimer son choix de manière anonyme afin que son vote reste secret. Hormis les votes par correspondances ${ }^{2}$, les procédures mises en œuvre doivent garantir le respect de la confidentialité en protégeant les électeurs des pressions, qu'il s'agisse de coercition ou d'achats de vote.

— dimension sociale

Un système de vote fait intervenir de nombreux acteurs : le dispositif technique destiné à recueillir les choix des électeurs, mais aussi, les électeurs eux-mêmes, les personnes chargées de sa gestion durant la période de vote et, le cas échéant, les personnes chargées de la maintenance et du dépannage du dispositif de vote.

\section{- dimension juridique}

La sincérité d'une élection est appréciée par un juge, en fonction du respect du code électoral et des textes applicables (décrets, arrêtés, etc.). En cas de contentieux électoral, si le juge évalue que les écarts avec la procédure ou des fraudes ou des erreurs ont été susceptibles d'avoir faussé l'élection en désignant un candidat plutôt qu'un autre (élection non sincère), il peut éventuellement annuler l'élection qui devra, par conséquent, se tenir de nouveau. En revanche, si les pièces portent sur un nombre insuffisant de voix pour changer l'issue de l'élection, le juge décide le plus souvent d'en valider le résultat tel quel.

- dimension politique

Les élections sont la procédure par laquelle les électeurs désignent leurs représentants élus. Détenteurs initiaux de la souveraineté dans un régime démocratique ${ }^{3}$, les électeurs, se défont volontairement de leurs pouvoirs (de police, de justice, etc.) afin de les confier à des élus qui les exerceront, directement ou en les déléguant, pendant la durée de leur mandat. La réussite de cette passation de pouvoir conditionne la légitimité des élus : un élu désigné au terme d'une procédure suscitant des forts soupçons de fraude verra sa légitimité contestée et ses décisions ne seront pas bien respectées. Les contestations peuvent aller jusqu'à prendre la forme d'émeutes ${ }^{4}$. Il est donc

2 Seul l'usage obligatoire d'un isoloir au sein d'un bureau de vote est à même de garantir la confidentalité.

3 Rappelons l'éthymologie du terme démocratie : $\delta \eta \mu \varsigma$ (peuple), $\kappa \rho \alpha \tau$ (gouvernement, pouvoir).

4 Les exemples sont légion: élection d'Ali Bongo au Cameroun en septembre 2009, élection de Mahmoud Ahmadinejad en Iran en juin 2009, élections législatives en Mongolie en juin 2008, élection de Mwai Kibaki au Kenya en décembre 2007, etc.

Enguehard, C. Introduction à l'analyse de chimères technologiques, le cas du vote électronique. Cahiers Droit Sciences et Technologie, $n^{\circ} 3$, p.261-278, CNRS editions, 2010. ISBN 978-2-271-06974-0. SSN 1967-0311. 
essentiel que les électeurs aient confiance dans la capacité du système électoral à désigner le candidat ayant obtenu le plus grand nombre de suffrages. La transparence permet aux électeurs de surveiller directement les opérations matérielles de vote et de fonder leur confiance sur leurs observations. À l'opposé, toute manipulation se déroulant loin des regards est susceptible de faire naître des rumeurs de fraude. Cet aspect fondamental des processus électoraux est incarné dans le mot "scrutin", synonyme d'élection, et provenant de la racine latine "scrutinium", mot qui a également engendré les mots "scruter" et "scrutateur".

\section{2 - Dispositifs techniques}

Le terme de vote électronique englobe une multitude de dispositifs techniques quelquefois désignés par plusieurs dénominations.

- Ordinateurs de Vote avec Bulletin Dématérialisé (OdV-BD) [Enguehard 2006] ou "machines à voter" ou "urnes électronique" : il s'agit d'ordinateurs, parfois d'une conception très simplifiée, conçus pour recueillir le choix exprimé par chaque électeur à l'aide d'une pression sur un bouton, d'un clic de souris, ou de la saisie d'un numéro. Les choix sont directement enregistrés en mémoire. À la fin de la période électorale, le système énonce le nombre de voix obtenues par chaque candidat.

- Ordinateurs de Vote avec Bulletin Matérialisé (OdV-BM) [Enguehard 2007] : il s'agit d'un aménagement des OdV-BD. Lorsqu'un électeur exprime un choix, un bulletin est imprimé. L'électeur peut vérifier sa correction à travers une vitre et le valider, le bulletin est alors déposé dans une urne à des fins d'éventuelles vérifications de la justesse du décompte automatique.

- Scanner : les bulletins des électeurs sont scannés par l'urne dans laquelle ils sont déposés ou ultérieurement lors de leur dépouillement centralisé. Le nombre de voix obtenues par chaque candidat est automatiquement énoncé par le scanner.

- Stylo numérique : les bulletins de vote sont imprimés sur un papier spécial permettant à la caméra installée dans le stylo numérique de se repérer. Les enregistrements réalisés par le stylo constituent une mémorisation des choix exprimés par les électeurs. Le bureau centralisateur dépouille ces enregistrements à l'aide du logiciel approprié.

- Vote par kiosque : les bureaux de vote sont équipés de terminaux reliés à un système centralisé. L'électeur s'identifie, s'authentifie, puis émet son choix, celui-ci est transmis au système centralisé.

- Vote par boîtier : une salle de délibération est équipée de boîtiers reliés à un ordinateur. Chaque électeur dispose d'un boîtier et exprime son choix par pression sur un bouton. L'ordinateur délivre les résultats.

- Vote par téléphone, Short Message Service (SMS) : l'électeur appelle une ligne réservée à la gestion du scrutin. Il s'identifie, s'authentifie puis vote au moyen des touches de son téléphone ou par l'envoi d'un message.

- Vote par internet [Enguehard 2008] ou "vote en ligne" ou "vote par correspondance électronique" : l'électeur se connecte sur un site de gestion du scrutin. Il s'identifie, s'authentifie puis émet son choix.

- Vote hybride [Enguehard 2008] : l'électeur s'exprime à l'aide d'une carte de vote comportant une marque qui l'identifie (numéro et code-barres ou image cryptée). Il fait son choix en cochant des cases ou en collant des étiquettes. Il envoie cette carte au centre de dépouillement. L'ensemble des cartes est automatiquement dépouillé par un scanner relié à une application informatique qui gère également les émargements.

Enguehard, C. Introduction à l'analyse de chimères technologiques, le cas du vote électronique. Cahiers Droit Sciences et Technologie, n³, p.261-278, CNRS editions, 2010. ISBN 978-2-271-06974-0. SSN 1967-0311. 


\begin{tabular}{|c|c|c|c|c|c|}
\hline & $\begin{array}{l}\text { environnement } \\
\text { contrôlé }^{5}\end{array}$ & $\begin{array}{l}\text { vote par } \\
\text { correspondance }^{6}\end{array}$ & $\begin{array}{l}\text { comptage } \\
\text { centralisé }\end{array}$ & $\begin{array}{l}\text { gestion des } \\
\text { émargements }^{7}\end{array}$ & $\begin{array}{l}\text { Matérialisation } \\
\text { des votes }\end{array}$ \\
\hline OdV-BD & oui & non & non & non & non \\
\hline OdV-BM & oui & non & non & non & oui \\
\hline Scanner & oui & non & non & non & oui \\
\hline Stylo numérique & oui & non & oui & non & oui \\
\hline Kiosque & oui & non & oui & oui & non \\
\hline Boîtier & oui & non & oui & $\begin{array}{l}\text { non (un électeur } \\
\text { par boîtier) }\end{array}$ & non \\
\hline Téléphone & non & oui & oui & oui & non \\
\hline Internet & non & oui & oui & oui & non \\
\hline Hybride & non & oui & oui & oui & oui \\
\hline
\end{tabular}

La brièveté de cet article ne nous permet pas de traiter de tous ces dispositifs. Notre propos sera concentré sur les OdV-BD et le vote par internet largement utilisés en France. L'analyse des OdVBD sera étendue aux formes de votes laissant une trace matérielle des votes : OdV-BM, mais aussi stylo numérique, scanner et vote hybride.

\section{2 - Ordinateurs de Vote avec Bulletin Dématérialisé (OdV-BD)}

\section{1 - Conception industrielle}

Les OdV-BD ont été spécifiés par les industriels comme des systèmes de traitement de l'information : les entrées que constituent les votes des électeurs sont collectées durant la journée de vote, le système restitue des résultats en exprimant le nombre de voix obtenues par chaque candidat ainsi que le nombre de votes nuls.

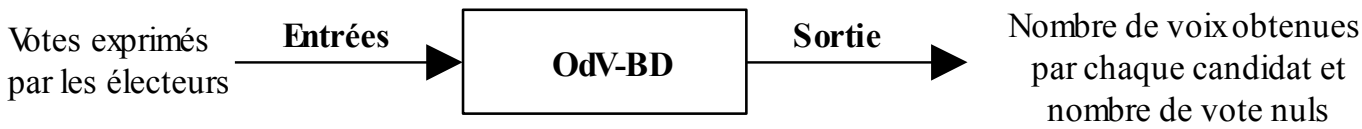

Système de vote électronique spécifié comme un système de traitement de l'information

Lors de l'usage d'un tel dispositif, les votes émis subissent des transformations qui échappent à la surveillance des électeurs. En effet, les choix sont exprimés par l'appui sur une touche. Cette force mécanique de quelques joules est convertie en une impulsion électrique elle-même transformée en

5 Un environnement contrôlé est un bureau de vote au sein duquel se déroulent les opérations électorales, en particulier, pour chaque électeur, vérification de l'identité, du droit à voter, signature de la liste d'émargement, choix effectué dans un isoloir (garant du respect de la confidentialité), enregistrement de chaque choix.

6 Pour les votes par correspondance, les électeurs reçoivent au préalable, par courrier, les identifiants et mots de passe qui leur permettront de s'identifier et de s'authentifier.

7 Lors de scrutins à distance, l'électeur n'étant pas en mesure de signer, le contrôle de l'unicité est limitée à la pose d'une marque sur le registre des émargements.

Enguehard, C. Introduction à l'analyse de chimères technologiques, le cas du vote électronique. Cahiers Droit Sciences et Technologie, n³, p.261-278, CNRS editions, 2010. ISBN 978-2-271-06974-0. SSN 1967-0311. 
une information codée en binaire susceptible de connaître d'autres traitements (cet enchaînement de modifications contraste avec la persistance des suffrages exprimés à l'aide de vrais bulletins lors de la procédure de vote traditionnelle).

En l'état actuel de la science, pour contrôler le fonctionnement d'une application informatique, il faut avoir un moyen d'en vérifier les résultats de manière indépendante, ou bien en suivre le déroulement pas à pas ${ }^{8}$.

D'une part, les entrées du système étant inconnues, il n'est pas possible de vérifier de manière indépendante que les résultats leur sont fidèles. Ni les scrutins précédents, ni les sondages effectués avant ou pendant la période de vote ne sont en mesure d'apporter une preuve incontestable de l'exactitude ou de la fausseté des résultats [Enguehard 2007b].

D'autre part, suivre l'exécution des programmes, instruction par instruction, depuis le choix émis par chaque électeur jusqu'au calcul final établissant les résultats, nécessite l'introduction de caméras pour examiner la saisie ainsi que de sondes logicielles dans les programmes. Outre la question de l'objectivité et de la neutralité de ces sondes et des programmes chargés d'analyser les données d'observation, l'analyse d'un journal de bord dans lequel sont notés et horodatés tous les événements ainsi que des images permettant de vérifier que les informations mémorisées correspondent bien aux choix exprimés par les électeurs aboutirait in fine au dévoilement des votes, en contradiction avec le principe d'anonymat.

Il existe donc une contradiction non résolue à ce jour entre l'exigence de transparence directe nécessaire à l'établissement de la confiance, le respect de l'anonymat, et la dématérialisation (suivie de transformations) des choix émis par les électeurs. Dès lors, ces systèmes de vote apparaissent comme des chimères techniques, prétendant marier des contraintes incompatibles. Nous verrons qu'il est même courant de leurs soient attribuées des capacités plus proches de la magie que du principe de réalité.

Une spécification complète des scrutins, prenant en compte leurs dimensions politiques, éthiques et sociales aurait fait émerger, entre autres, le rôle de scrutateur dévolu à tout électeur. Au contraire, la spécification mono-dimensionnelle a restreint les électeurs à n'être que des émetteurs de données. Il est d'ailleurs significatif que les scrutateurs aient disparu des bureaux de vote équipés de ces dispositifs.

\section{2 - De la mécanique à l'informatique}

En France, les Ordinateurs de Vote avec Bulletin Dématérialisé sont désignés dans le code électoral par le terme "machines à voter". Leur usage est autorisé par l'article L57-19 complété par un règlement technique [Intérieur 2003] fixant les conditions d'agrément de ces dispositifs.

Lors de la promulgation de la loi, en 1969, le terme "machine à voter" désignait des systèmes mécaniques, fonctionnant sans électricité, chaque vote exprimé par une pression sur un bouton entraînant une roue dentée qui ajoute une unité sur un compteur de type odomètre mécanique. Peu déployées (600 exemplaires), ces machines de marque Alcatel ont été abandonnées en 1988.

Il existe un saut technologique entre les machines à voter mécaniques et les "machines à voter" informatiques apparues avec le règlement technique de 2003 :

- Un dispositif mécanique fonctionne selon un schéma fixe conditionné par la forme et la disposition des pièces qui le composent et régi par les lois de la physique classique. La complexité

\footnotetext{
8 La démarche de tests ou de preuve de programme rencontre, entre autres, le problème non trivial d'identité entre les programmes examinés et ceux qui sont effectivement en service.

9 Loi n69-419 du 10 mai 1969.
}

Enguehard, C. Introduction à l'analyse de chimères technologiques, le cas du vote électronique. Cahiers Droit Sciences et Technologie, n³, p.261-278, CNRS editions, 2010. ISBN 978-2-271-06974-0. SSN 1967-0311. 
d'un système mécanique pourrait être estimée par le nombre des pièces qui le composent et qui sont susceptibles de se mouvoir. Dans le cas d'une machine à voter elle est limitée à quelques centaines de pièces. Il n'y a pas de mémoire. Toutes les pièces sont d'une taille suffisante pour être visibles par un œil humain.

Une machine à voter mécanique est un compteur du nombre de pressions exercées sur une touche (à chaque touche est associée un compteur).

- Un dispositif informatique héberge une mémoire dans laquelle sont stockées des informations. Le comportement du dispositif dépend de programmes, qu'ils soient utilisés directement ou indirectement $^{10}$, et du contenu de la mémoire. Un tel dispositif est non stable : modifier un programme suffit à en modifier le comportement. La complexité d'un système informatique peut être mesurée par le nombre d'instructions composant les programmes et par le nombre de données contenues dans sa mémoire. Dans le cas d'un ordinateur de vote, elle peut être évaluée à plusieurs dizaines de milliers d'instructions et plusieurs milliers d'emplacements mémoires pour stocker les valeurs des variables utilisées dans les programmes et les votes eux-mêmes. Cette complexité a pour conséquence de donner au dispositif la capacité d'adopter des comportements différents selon les situations rencontrées. De plus, l'activité d'un ordinateur de vote, dépendante des mouvements d'électrons invisibles à l'œil nu, n'est pas directement observable.

Un ordinateur de vote simule le fonctionnement d'un ensemble de compteurs du nombre de pressions exercées sur ses touches (les touches elles-mêmes peuvent être réelles ou représentées sur l'écran).

Avec une machine mécanique, l'électeur agit directement (par une action mécanique) sur le compteur des voix. En revanche, avec une machine électronique, c'est un ensemble de logiciels qui interprète les interactions avec chaque électeur puis exprime le comptage des suffrages.

La distinction entre un objet réel et sa représentation est essentielle. Elle a été illustrée avec brio par René Magritte dans son œuvre intitulée fort justement "La trahison des images". La représentation très réaliste d'une pipe est légendée par la phrase "Ceci n'est pas une pipe. ». Effectivement, il est impossible de mettre du tabac dans la pipe représentée, de l'allumer et d'en tirer quelques bouffées. En revanche, il est possible de décrocher cette toile, d'en ôter le cadre, et de la rouler, ce que ne supporterait pas une pipe en bois. Les propriétés d'une pipe sont donc, par essence, différentes de celles de la représentation d'une pipe.

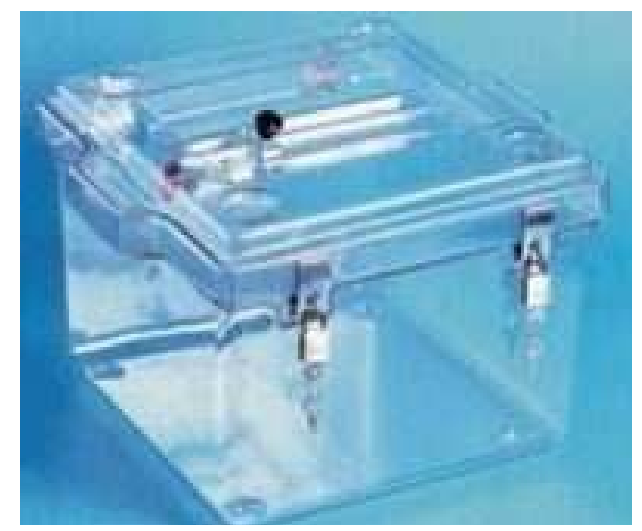

Ceci n'est pas une urne [Appel 2006]

10 Système d'exploitation, compilateur extérieur éventuellement utilisé pour créer le programme exécutable présent sur le dispositif de vote à partir d'un programme écrit par un informaticien, etc.

Enguehard, C. Introduction à l'analyse de chimères technologiques, le cas du vote électronique. Cahiers Droit Sciences et Technologie, n³, p.261-278, CNRS editions, 2010. ISBN 978-2-271-06974-0. SSN 1967-0311. 
De la même façon que l'image d'une urne ci-dessus n'est pas une urne, la représentation virtuelle d'un compteur au sein d'un ordinateur n'est pas un compteur. Les différences d'avec un vrai compteur sont fondamentales. Par exemple, un compteur mécanique peut être conçu de manière à être restreint à additionner des éléments un à un ou à être remis à zéro et afin que toute soustraction soit physiquement impossible. Un ordinateur possède, potentiellement, un comportement infiniment modulable. Restreindre les capacités d'un ordinateur à un ensemble de tâches limitées (additionner, ne pas soustraire), et être capable de le prouver, nécessite de savoir contrôler l'ensemble des programmes qu'il héberge. Il s'agit d'un problème non trivial pour lequel il n'existe pas de solution satisfaisante ${ }^{11}$.

Considérer les représentations d'objets comme si elles possédaient les mêmes propriétés que les objets eux-mêmes constitue donc une erreur méthodologique grave.

\section{3 - Cadre juridique}

Voici quelques exemples de textes officiels régissant les OdV-BD. Ils ont été choisis en fonction de leur écart avec le principe de réalité.

— L'article L63 du code électoral dispose :

"Dans les bureaux de vote dotés d'une machine à voter, le bureau de vote s'assure publiquement, avant le commencement du scrutin, que la machine fonctionne normalement et que tous les compteurs sont à la graduation zéro. »

Tester avec succès le fonctionnement d'un dispositif électronique à un moment donné ne peut constituer une preuve de son bon fonctionnement à tout instant ; du fait de la complexité des systèmes, les tests ne peuvent être exhaustifs, cette procédure est donc inadéquate.

Il n'y a pas de compteur gradué sur les OdV-BD agréés en France.

Cet article de loi, en vigueur depuis 1991, avait été écrit pour les machines à voter mécaniques. Les législateurs n'ont pas jugé nécessaires de l'amender lors de l'apparition des OdV-BD.

— Le 12 février 2005, la loi L57-1 du code électoral est enrichie d'un nouvel alinéa ${ }^{12}$ :

"[Les machines à voter doivent] permettre aux électeurs handicapés de voter de façon autonome, quel que soit leur handicap ; "

Des études d'accessibilité des OdV-BD ont montré que de nombreux électeurs, non handicapés, ne parviennent pas à voter pour le candidat de leur choix. Les difficultés s'accroissent pour les exclus de la technologie ainsi que pour les mal-voyants, les aveugles et les personnes âgées qui doivent se faire assister, perdant ainsi le bénéfice de la confidentialité. Finalement, il apparaît

11 «If you have a computer sitting right in front of you, you can ask it to print out the programs installed its hard drive — but you are asking a computer program installed on the machine to read the hard drive, and you don't know whether that computer program is telling the truth. You can open up the machine and remove the hard drive, to read it from another computer that you trust - that way you know what's on the hard drive, but you don't know whether the software in the BIOS of the computer (which is elsewhere in the box than the hard drive) is actually running the program from the hard drive, or is running another program entirely. » [Appel 2006]

traduction: «Si vous avez un ordinateur juste en face de vous, vous pouvez lui demander d'imprimer tous les programmes installés sur son disque dur — mais c'est en fait demander à un programme informatique installé sur la machine de lire le disque dur, et vous ne saurez pas si ce programme informatique dit la vérité. Vous pouvez ouvrir la machine et enlever son disque dur afin de le lire depuis un autre ordinateur en lequel vous avez confiance - de cette façon, vous saurez ce qu'il y a sur le disque dur. Par contre, vous ne saurez pas si le logiciel dans le BIOS de l'ordinateur (qui se situe ailleurs que le disque dur) fait réellement tourner le programme depuis le disque dur, ou bien un tout autre programme. »

12 Modification issue de la loi n²005-102 du 11 février 2005 pour l'égalité des droits et des chances, la participation et la citoyenneté des personnes handicapées.

Enguehard, C. Introduction à l'analyse de chimères technologiques, le cas du vote électronique. Cahiers Droit Sciences et Technologie, n³, p.261-278, CNRS editions, 2010. ISBN 978-2-271-06974-0. SSN 1967-0311. 
que l'usage de la technologie constitue un obstacle pour de nombreux électeurs [Michel \& al. 2007].

Cet alinéa révèle un fantasme attaché à la technologie imaginée comme toute-puissante, jusqu'à être capable de remédier à tous les handicaps.

— En 2003, le Ministère de l'Intérieur, organisateur des élections politiques, publie un règlement technique sous forme d'un décret [Intérieur 2003]. Ce règlement énumère 18 principes à respecter et 114 exigences que doivent satisfaire les dispositifs, et détaille l'organisation de la procédure d'agrément que les OdV-BD doivent passer avec succès pour être utilisés : chaque modèle fait l'objet d'une inspection par un organisme de certification.

Deux des principes à respecter et une exigence attirent particulièrement notre attention :

" caractère vérifiable : les résultats du vote peuvent être vérifiés après le dépouillement du scrutin "

La procédure de vérification prévoit que le dispositif de vote émette une seconde édition des résultats qu'il a déjà énoncés. Cette procédure n'a aucune validité scientifique : une procédure de vérification des résultats doit être indépendante du processus par lequel le résultat a été établi [NIST 2006].

" transparence : le processus doit pouvoir être examiné et vérifié »

Outre le fait que l'examen et la vérification du processus sont, légalement, hors de portée des électeurs pour cause de protection du secret industriel et commercial et de la sécurité des élections, il n'existe actuellement aucune procédure scientifique d'examen ou de test capable de déceler sûrement les erreurs ou les malfaçons dans des programmes.

«Exigence 3 :

Afin de vérifier l'exactitude des données mémorisées dans la machine à voter, leur affichage et leur impression doivent être possibles avant l'ouverture du scrutin. »

L'exactitude de données mémorisées ne peut être vérifiées par leur impression ou leur affichage. Un ordinateur peut imprimer une version d'une donnée et en détenir une autre en mémoire.

L'examen du règlement technique laisse donc apparaître une méconnaissance des principes scientifiques en général, et de la science informatique en particulier.

Il est surprenant que les rapports fournis par les organismes d'inspection aient pu aboutir quand même à des délivrances d'agréments. Une procédure juridique [Graton 2007] a révélé que, pour au moins un des trois modèles agréés en France, l'inspection menée avait évalué le respect de critères de manière très approximative.

L'Organisation pour la Sécurité et la Coopération en Europe, à la suite de sa mission d'observation de l'élection présidentielle 2007, a d'ailleurs relevé : « il est préoccupant que les organismes agréés de vérification aient un pouvoir discrétionnaire aussi important pour apprécier la marge de variation acceptable pour la validation de chaque critère et pour déterminer si certains critères sont pertinents ou non. » [OSCE 2007].

Il apparaît donc que les législateurs, juristes et conseillers d'État ayant élaboré les outils juridiques accompagnant les OdV-BD en France ont continué à raisonner comme s'il s'agissait de dispositifs mécaniques et ont ignoré les nouvelles disciplines qu'il convenait de prendre en compte. Ils ont imaginé des procédures n'ayant aucune validité scientifique, révélant parfois une confusion entre le monde réel et le monde virtuel et trahissant une intériorisation profonde des discours commerciaux parant les ordinateurs de vertus imaginaires (sécurité et fiabilité totales, utilisabilité parfaite, etc.).

Enguehard, C. Introduction à l'analyse de chimères technologiques, le cas du vote électronique. Cahiers Droit Sciences et Technologie, n³, p.261-278, CNRS editions, 2010. ISBN 978-2-271-06974-0. SSN 1967-0311. 


\section{3 - Dispositifs de vote électronique avec trace "papier"}

\section{1 - Genèse des Ordinateurs de Vote avec Bulletin Matérialisé}

Aux États-Unis, un consensus alliant scientifiques et organisateurs des élections s'est établi autour du caractère invérifiable des résultats des OdV-BD [NIST 2006]. Un nouveau concept de dispositif visant à produire des traces matérielles des votes tout en préservant l'anonymat a émergé : les Ordinateurs-de-Vote avec Bulletin Matérialisé [Mercuri 2002] ${ }^{13}$. Ces dispositifs équipent plus de la moitié des états des États-Unis et l'ensemble des bureaux de vote du Vénézuela.

Puisqu'un dysfonctionnement d'un ordinateur de vote peut rester invisible du fait de la concomitance de la perte de transparence, du respect de l'anonymat et de la dématérialisation des données, l'idée a émergé de rematérialiser les bulletins à des fins d'éventuelles vérifications ultérieures.

Deux points de vérification ont été définis :

1 - l'électeur vérifie que le bulletin matérialisé correspond bien aux choix ${ }^{14}$ qu'il a exprimés ;

2 - les bulletins matérialisés sont collectés dans une urne qui peut être dépouillée manuellement afin de vérifier la justesse des résultats émis par le dispositif ${ }^{15}$.

Ces deux points de contrôle sont destinés à détecter des dysfonctionnements qui peuvent avoir pour conséquence le décompte erroné des voix exprimées.

\section{2 - Confrontation avec la réalité juridique}

\section{Premier point de contrôle}

Le respect du principe de confidentialité astreint chaque électeur à voter seul et à ne collecter aucune preuve de vote (enregistrement vidéo par exemple) susceptible de l'exposer à des pressions ou à la tentation de vendre son vote. Par conséquent, un électeur qui détecte une divergence entre le bulletin imprimé et les choix qu'il a exprimés ne peut en apporter de preuve juridique et ne peut produire aucun témoignage d'un tiers corroborant ses dires.

Il apparaît donc que le premier point de contrôle est légalement inopérant.

De plus, l'observation d'élections menées avec ces dispositifs et la réalisation d'études ergonomiques ont montré que plus d'un tiers des électeurs ne détectent pas les modifications affectant les choix qu'ils ont exprimés [Campbell \& al. 2009]. Il est courant, au moment du recompte, de constater que des bulletins sont illisibles ou manquants. Dans ce cas, il est procédé à une impression des bulletins mémorisés par le dispositif de vote qu'il s'agit de contrôler et ces bulletins sont recomptés bien qu'ils n'aient pas été vérifiés par les électeurs.

\section{Second point de contrôle}

Le second point de contrôle est optionnel. Seuls quelques dispositifs de vote sont désignés pour voir leurs résultats vérifiés (par exemple 1\% en Californie). Les critères de désignation dépendent des législations en vigueur. On peut choisir de recompter les bulletins des bureaux de vote présentant des résultats serrés, ou sélectionner au hasard un certain pourcentage d'urnes à vérifier, ou encore ne

13 Les scanners, stylos numériques et le vote par correspondance hybride constituent aussi des dispositifs laissant des traces permettant des vérifications.

14 Aux États-Unis et au Vénézuéla, l'électeur s'exprime couramment sur plusieurs questions.

15 Ce second point de contrôle pourraît, théoriquement, être déployé pour toutes les technologies de vote fondées sur l'usage de bulletins matérialisés : stylo numérique, scanner, vote par correspondance hybride.

Enguehard, C. Introduction à l'analyse de chimères technologiques, le cas du vote électronique. Cahiers Droit Sciences et Technologie, n³, p.261-278, CNRS editions, 2010. ISBN 978-2-271-06974-0. SSN 1967-0311. 
procéder à des vérifications qu'en cas de demandes formelles des candidats ou de contentieux électoraux, etc.

Dans tous les cas, le contenu des urnes est déplacé et stocké jusqu'au déclenchement éventuel de la procédure de recompte. La surveillance effective des bulletins par les électeurs, initiée dans les bureaux de vote, ne peut alors plus s'exercer. Il s'agit d'une rupture irrémédiable qui a pour conséquence la perte de la transparence qu'il s'agissait justement de rétablir.

La procédure de comptage éventuellement mise en œuvre n'est pas toujours publique.

En cas de différence entre le résultat énoncé par le dispositif et le comptage manuel, le comptage manuel fait officiellement foi mais, il n'y a, à notre connaissance, aucune législation dans les pays où sont utilisés ces dispositifs pour extrapoler les divergences observées à l'ensemble des dispositifs de vote qui n'ont pas bénéficié de vérification (en Californie, par exemple, il s'agit de $99 \%$ des dispositifs en service). Leurs résultats sont donc considérés comme justes bien qu'ils n'aient fait l'objet d'aucune vérification.

Les dispositifs présentant des pannes matérielles peuvent être ponctuellement réparés ou réformés mais il n'existe pas de procédure systématique exigeant recherche et correction des erreurs logicielles, et production d'une nouvelle version des programmes, peut-être à cause de la complexité, de la durée et du coût qu'entrainerait une demande d'agrément pour cette nouvelle version du dispositif de vote.

\section{Bilan}

Lors de la spécification de ce nouveau système de vote les informaticiens ont raisonné en ne tenant compte que de la sphère technique dans laquelle ils évoluent. Ils sont habitués, à ce que la détection d'une anomalie donne lieu à un signalement. Ensuite, la recherche de la cause de l'anomalie, puis la correction des programmes, conduisent à la production de nouvelles versions ou à la distribution de rustines logicielles permettant aux usagers de mettre à jour les programmes dont ils disposent. Comme nous venons de le voir, ce schéma ne prenant pas en compte les aspects juridiques organisationnels d'un scrutin $\mathrm{a}$, une fois de plus, conduit à la production d'un système de vote inadéquat.

Finalement, il apparaît que la rematérialisation des bulletins a introduit des faiblesses par l'adjonction d'imprimantes susceptibles de tomber en panne, ou de manquer d'encre ou de papier, et a complexifié l'acte de voter pour les électeurs. La procédure de recompte manuel n'est pas appropriée pour détecter avec succès les dysfonctionnements et ne peut être considérée comme une vérification. Le dispositif ne rétablit pas la transparence et se révèle juridiquement non opérationnel.

\section{3 - Vote par correspondance hybride}

En France, le seul mode de vote électronique permettant un recompte manuel des bulletins est le vote par correspondance hybride. Il n'existe aucune procédure légale permettant d'obtenir une vérification immédiate du décompte automatique. Seul un juge électoral est en droit de demander ce recompte dans le traitement d'un recours en contentieux électoral, encore faudrait-il lui présenter des preuves assez convaincantes pour qu'il prenne une telle décision.

\section{4 - Au-delà des ordinateurs de vote, le vote par internet}

La brièveté de cet article ne permet pas de développer une étude complète du vote par internet, seuls quelques points saillants complétant la démonstration précédente seront relevés ici.

Le vote par internet a connu un développement sans précédent ces dernières années. Huit décrets,

Enguehard, C. Introduction à l'analyse de chimères technologiques, le cas du vote électronique. Cahiers Droit Sciences et Technologie, n³, p.261-278, CNRS editions, 2010. ISBN 978-2-271-06974-0. SSN 1967-0311. 
cinq arrêtés et une ordonnance ${ }^{16}$ organisent son usage légal selon des modalités diverses (par exemple, l'usage du vote par internet est autorisé dans le cas des votes professionnels, mais il est imposé pour l'élection des conseils de l'ordre des infirmiers).

Ce mode de vote présente des caractéristiques proches des ordinateurs de vote :

- représentation virtuelle des objets du vote : bulletins, urne, enveloppes (auxquels s'ajoute le registre d'émargement) ;

- assemblage de propriétés inconciliables : transparence, anonymat des votes et dématérialisation des choix émis par les électeurs.

Il présente en plus la particularité d'être une des formes du vote par correspondance. Comme tous les systèmes de vote par correspondance, les systèmes de vote par internet reçoivent les votes des électeurs accompagnés de leur identité. Ces informations permettent de tenir à jour un registre des émargements afin de respecter le principe de l'unicité (un électeur, une voix). Garantir le respect de l'anonymat dans ces circonstances constitue un problème scientifique de haute volée, non résolu à ce jour, et donnant lieu à d'intenses recherches ([Chatzikokolakis \& al. 2008] par exemple).

Les votes émis par les électeurs étant considérés comme des données personnelles, la Commission Nationale de l'Informatique et des Libertés (CNIL) s'est saisie de la question du vote par internet dont elle a, au passage, apprécié la nature opaque ${ }^{17}$. Elle a préconisé des dispositions techniques [CNIL 2003] visant à garantir le respect de l'anonymat. Ces recommandations tendent essentiellement à complexifier la violation de l'anonymat mais ne peuvent l'interdire : les textes juridiques n'ont pas la capacité de décréter des résultats scientifiques. Finalement, la CNIL a produit une procédure légale de validation des systèmes de vote par internet alors qu'il est impossible de vérifier que ses préconisations ont été effectivement suivies et qu'elles sont efficaces, même en ayant recours à une expertise.

L'examen de deux textes officiels dénote des habituelles confusions et approximations.

«Le comité technique d'organisation des élections peut demander la communication du procès-verbal établi par l'autorité administrative attestant que la liste d'émargement est vierge, que les urnes électorales sont vides [...]. » (arrêté du 13 mars 2008).

L'autorité administrative n'est pas en mesure de faire les constats qu'elle est censée reporter sur le procès-verbal car il n'y a ni liste d'émargement, ni urne électorale, mais des représentations de ces objets dans la mémoire d'un ordinateur.

Ces objets virtuels sont dotés de propriétés nouvelles qui invalident les mesures habituelles. Ainsi, avec le vote traditionnel, la vérification de la vacuité de l'urne au début de la période de vote, puis le contrôle des modifications apportées à son contenu (limitées à l'ajout des votes des

16 - élection des membres du Conseil national des barreaux (décret n²002-1306)

— élection des membres de l'Assemblée des Français de l'Étranger - cette assemblée de 79 élus élit 12 sénateurs (auxquels s'ajouteront 11 députés en 2012 ) parmi ses membres - (décrets n²003-396, 2006-285, 2009-525, arrêtés du 6 avril 2006, des 11 et 13 mai 2009).

- élections professionnelles : délégués du personnel et représentants du personnel au comité d'entreprise (décret $\left.\mathrm{n}^{\circ} 2007-602\right)$

— élections prud'homales de 2008 à Paris (ordonnance ${ }^{\circ}$ 2004-603, décret n²007-1130, arrêté du 21 juillet 2008)

— élections des conseils de l'ordre des infirmiers (décrets n²007-552, 2007-554, arrêté du 13 mars 2008)

17 « Le vote manuel a comme principale qualité sa grande simplicité permettant à l'électeur, s'il le souhaite, à tout moment de vérifier facilement la régularité du déroulement d'un scrutin.

Force est de constater qu'il n'en est pas de même pour les systèmes de vote électronique comme la Commission a pu maintes fois le vérifier à l'occasion de l'instruction des dossiers. » [CNIL RA 2003]

Enguehard, C. Introduction à l'analyse de chimères technologiques, le cas du vote électronique. Cahiers Droit Sciences et Technologie, n³, p.261-278, CNRS editions, 2010. ISBN 978-2-271-06974-0. SSN 1967-0311. 
électeurs autorisés) sont des mesures classiques de protection de son intégrité car une urne est un dispositif inerte, incapable de modifier les votes qu'elle contient. Bien qu'imparfaites, leur mise en œuvre a la capacité de faire échouer ou de révéler des atteintes affectant un grand nombre de suffrages et susceptibles d'altérer la sincérité des résultats [Enguehard 2008]. En revanche, le dispositif qui héberge une urne virtuelle est en constante modification, passant d'un état à un autre à chaque instant, et les modifications du contenu d'une urne virtuelle sont le résultat de déplacements d'électrons qu'il est impossible d'observer directement. Les mesures de surveillance et contrôle qui peuvent être mises en œuvre ne peuvent s'exercer que sur une image de la représentation virtuelle de l'urne (affichage sur un écran par exemple, ou impression d'un ticket), image construite par le système qu'il s'agit de surveiller et dont il est impossible de vérifier la justesse.

Il apparaît que des procédures conçues pour s'appliquer à objets réels ont été décalquées pour accompagner les objets virtuels sans que leur validité ne soit interrogée.

"Les fichiers comportant les éléments d'authentification des électeurs, les clés de chiffrement et de déchiffrement et le contenu de l'urne ne doivent être accessibles qu'aux personnes chargées de la gestion et de la maintenance du système. " (décret n²007-602).

Par ce texte, les personnes en charge de l'organisation technique peuvent légalement accéder à des informations permettant de lever l'anonymat des votes et d'atteindre à la sincérité des élections [Graton 2007b].

Comme les ordinateurs de vote, le vote par internet est affublé de qualités imaginaires. Ainsi, le Centre National de la Recherche Scientifique (CNRS), organisateur d'un vote par internet pour le renouvellement des membres de son Conseil d'Administration en juin 2009, a-t-il informé ses électeurs que ce mode de vote respecte « la confidentialité, le secret du vote : permettant d'exercer son droit de vote sans pression extérieure $\rangle^{18}$. Or, les électeurs, votant via internet depuis n'importe quel ordinateur connecté à la toile mondiale, ne bénéficient pas de la protection d'un isoloir au sein d'un bureau de vote. Ils sont donc susceptibles, comme lors de tout vote par correspondance, d'être soumis à des pressions (de leur famille, leur supérieur hiérarchique, leurs collègues, etc.).

Cet exemple est caractéristique des propriétés magiques associées à l'usage des nouvelles technologies.

\section{5 - Conclusion}

L'organisation d'une élection concerne de nombreux intervenants : les candidats, les électeurs, les organisateurs des élections, mais aussi les juges qui statueront sur sa régularité et les scrutateurs et délégués des candidats qui veillent à détecter et signaler les anomalies. Il apparaît que les objets du vote doivent également être considérés comme des acteurs à part entière. Leur métamorphose en des représentations virtuelles a modifié leur essence (réelle/électronique) et, par conséquent, les propriétés qui leurs sont attachées. Elle a aussi profondément affecté le rôle des humains. Les électeurs sont devenus émetteurs de données, les scrutateurs ont disparu, les organisateurs des élections ont perdu leur pouvoir de contrôle des opérations affectant les votes pour se cantonner au rôle de serviteur du dispositif électronique en place ; ils doivent, comme les délégués, se contenter des indices fournis par un système dont ils n'ont pas la maîtrise.

Nous avons constaté que de nombreux textes légaux régissant les élections, bien que récents, ont été rédigés en ignorant les nouvelles propriétés de ces objets virtuels, comme si cette transformation

18 http://www.sg.cnrs.fr/elections/old/CA/voteparinternetv3.htm

Enguehard, C. Introduction à l'analyse de chimères technologiques, le cas du vote électronique. Cahiers Droit Sciences et Technologie, $n^{\circ} 3$, p.261-278, CNRS editions, 2010. ISBN 978-2-271-06974-0. SSN 1967-0311. 
n'avait aucune conséquence. Ce constat entraîne une interrogation immédiate. Pourquoi les juristes se sont-ils contentés d'imiter les anciennes procédures jusqu'à les transformer en une liturgie absconse, plutôt que de construire un environnement juridique adapté à la nouvelle nature du vote électronique et susceptible d'installer le sentiment de sécurité et de confiance indispensable à la tenue d'élections sereines?

Il apparaît que cette impossibilité à construire des textes adaptés aux nouveaux dispositifs de vote est une conséquence directe de la nature chimérique du vote électronique, assemblage de caractéristiques incompatibles entre elles (transparence directe, respect de l'anonymat et dématérialisation des votes) que vient logiquement parachever un encadrement juridique inadapté jusqu'à l'absurde. Les évolutions récentes confirment cette tendance : le ministère de l'intérieur invite maintenant les mairies à mettre en scène une configuration publique des interfaces des dispositifs électroniques de votes qu'ils détiennent [Intérieur 2008], des huissiers signent des constats censés garantir l'anonymat de procédures de vote par internet.

On ne s'étonnera dès lors plus beaucoup des capacités fabuleuses fréquemment prêtées à ces chimères modernes en contradiction avec les travaux scientifiques ou les observations portant sur les expériences antérieures : faculté des handicapés à voter seuls quel que soit leur handicap, protection contre les pressions lors d'un vote à distance, mais aussi, augmentation certaine du taux de participation.

\section{Références}

[Appel 2006] Appel, W. A. Ceci n'est pas une urne: On the Internet vote for the Assemblée des Français de l'Etranger. juin 2006.

[Campbell \& al. 2009] Campbell, B. A. \& Byrne, M. D. Now Do Voters Notice Review Screen Anomalies? A Look at Voting System Usability. EVT/WOTE'09, Electronic Voting Technology Workshop / Workshop on Trustworthy Elections, Montreal, Canada, August 10-11, 2009.

[Chatzikokolakis \& al. 2008] Chatzikokolakis, K. Palamidessi, C., Panangaden, P. Anonymity Protocols as Noisy Channels. Information and Computation, 206: 378-401, 2008.

[CNIL 2003] Commission Nationale de l'Informatique et des Libertés. Délibération $n^{\circ}$ 03-036 portant adoption d'une recommandation relative à la sécurité des systèmes de vote électronique. 1er juillet 2003.

[CNIL RA 2003] Commission Nationale de l'Informatique et des Libertés. Rapport d'activité. 2003.

[Graton 2007] Graton, J.-D. Référé-liberté de Géraldine Carayol. Vaucresson, 21 avril 2007.

[Graton 2007b] Graton, J.-D. Recours c/Ministre des affaires sociales et du travail. 26 juin 2007.

[Enguehard 2006] Enguehard, C. Le vote électronique en France: opaque \& invérifiable. Legalis.net, \#4, p.83-97, décembre 2006.

[Enguehard 2007] Enguehard, C. Vote électronique et preuve papier. Actes du 14 ème Colloque international "De l'insécurité numérique à la vulnérabilité de la société". Paris, 14 et 15 Juin 2007.

[Enguehard 2007b] Enguehard, C. Éléments de réflexion. Participation aux travaux du groupe de travail sur les machines à voter du Ministère de l'intérieur, de la sécurité intérieure et des libertés locales. 30 novembre 2007.

[Enguehard 2008] Enguehard, C. Analyse des vulnérabilités de trois modes de vote à distance. Legalis.net, p.13-31, \#3, septembre 2008.

[Intérieur 2003] Ministère de l'intérieur, de la sécurité intérieure et des libertés locales. Règlement technique fixant les conditions d'agrément des machines à voter. Annexe à l'arrêté du 17 novembre 2003. NOR : INTX0306924A, 17 novembre 2003. 
[Intérieur 2008] Ministère de l'intérieur, de l'outre-mer et des collectivités territoriales. Utilisation des machines à voter à l'occasion des élections municipales et cantonales des 9 et 16 mars 2008. NORINTA0800023C, 1er février 2008.

[Mercuri 2002] Mercuri, Rebecca. A Better Ballot Box? IEEE Spectrum Online, October 2002.

[Michel \& al. 2007] Michel, G., De Abreu, W. \& Brangier, É. Electoral Ergonomic Guidelines to Solve the Interference of new Technologies and the Dangers of their Broader use in Computerised Voting. 7th European Conference on e-Government, p.337-348, Den Haag, Netherlands, 21-22 June 2007.

[NIST 2006] National Institute of Standards and Technology. Requiring Software Independence in VVSG 2007: STS Recommendations for the TGDC. November 2006.

[Offerlé 2002] Offerlé, Michel. Un homme, une voix ? Histoire du suffrage universel. Gallimard, Collection Découvertes Gallimard, ISBN : 2-07-076406-0 (br.), 2002.

[OSCE 2007] Organisation pour la Sécurité et la Coopération en Europe / Bureau des Institutions Démocratiques et des Droits de l'Homme. France - élection présidentielle 22 avril et 6 mai 2007. Rapport de la Mission d'évaluation électorale, 4 October 2007.

Note : la version publiée de cet article est illustrée par la "Chimère aux seins de l'Artémis d'Ephèse" de Gustave Moreau (1826-1898) (C) Photographie RMN - René-Gabriel Ojéda. 